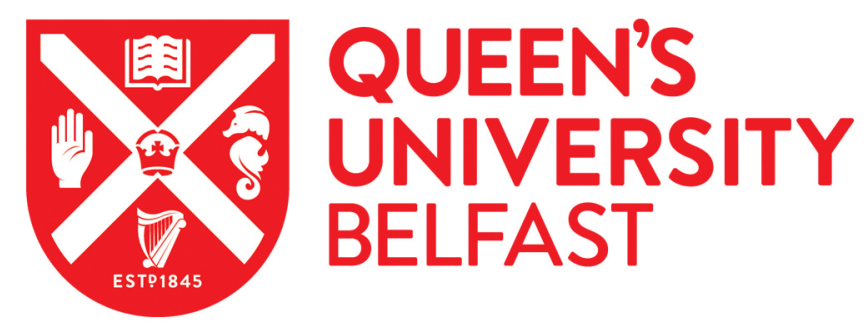

\title{
Optimizing Differentiated Discretization for Audio Circuits Beyond Driving Point Transfer Functions
}

Germain, F. G., \& Werner, K. J. (2017). Optimizing Differentiated Discretization for Audio Circuits Beyond Driving Point Transfer Functions. In Proceedings of the IEEE Workshop on Applications of Signal Processing to Audio and Acoustics (WASPAA) (pp. 1-5). https://doi.org/10.1109/WASPAA.2017.8170060

Published in:

Proceedings of the IEEE Workshop on Applications of Signal Processing to Audio and Acoustics (WASPAA)

Document Version:

Peer reviewed version

Queen's University Belfast - Research Portal:

Link to publication record in Queen's University Belfast Research Portal

Publisher rights

(c) 2017 IEEE.

This work is made available online in accordance with the publisher's policies. Please refer to any applicable terms of use of the publisher.

\section{General rights}

Copyright for the publications made accessible via the Queen's University Belfast Research Portal is retained by the author(s) and / or other copyright owners and it is a condition of accessing these publications that users recognise and abide by the legal requirements associated with these rights.

Take down policy

The Research Portal is Queen's institutional repository that provides access to Queen's research output. Every effort has been made to ensure that content in the Research Portal does not infringe any person's rights, or applicable UK laws. If you discover content in the Research Portal that you believe breaches copyright or violates any law, please contact openaccess@qub.ac.uk. 


\section{OPTIMIZING DIFFERENTIATED DISCRETIZATION FOR AUDIO CIRCUITS BEYOND DRIVING POINT TRANSFER FUNCTIONS}

\author{
François G. Germain
}

Center for Computer Research in Music and Acoustics
Stanford University, Stanford, CA
francois @ ccrma.stanford. edu
Kurt James Werner

\author{
Sonic Arts Research Centre \\ Queen's University Belfast, Belfast, UK \\ $\mathrm{k}$. werner@qub. ac.uk
}

\begin{abstract}
One goal of Virtual Analog modeling of audio circuits is to produce digital models whose behavior matches analog prototypes as closely as possible. Discretization methods provide a systematic approach to generate such models but they introduce frequency response error, such as frequency warping for the trapezoidal method. Recent work showed how using different discretization methods for each reactive element could reduce such error for driving point transfer functions. It further provided a procedure to optimize that error according to a chosen metric through joint selection of the discretization parameters. Here, we extend that approach to the general case of transfer functions with one input and an arbitrary number of outputs expressed as linear combinations of the network variables, and we consider error metrics based on the $L^{2}$ and the $L^{1}$ norms. To demonstrate the validity of our approach, we apply the optimization procedure for the response of a Hammond organ vibrato/chorus ladder filter, a 19-output, 36th order filter, where each output frequency response presents many features spread across its passband.
\end{abstract}

Index Terms - Discretization schemes, Virtual Analog modeling, Optimization, Bilinear transform, Audio effects

\section{INTRODUCTION}

One of the main methods available to simulate physical systems such as audio circuits, where the governing equations are available, is through the use of discretization methods $[1,2]$. For audio circuits, the governing equations can often be derived from the available schematics using circuit analysis methods [3]. In the virtual analog context [4], different formalisms for discretization are used, including wave digital filters [5-8] and state-space models $[9,10]$. However the underlying discretization methods are largely identical, and systems generated using those methods present similar distortion in their response compared to the original system. In the case of linear time-invariant systems, that distortion manifests itself as an error in the system frequency response. A typical example of such distortion is the frequency warping introduced by the trapezoidal method (i.e., the standard bilinear transform) [11,12].

Typical applications of discretization methods use a single method across the entire system to be modeled. Typical methods include methods from the Möbius transform family, e.g., the forward Euler method, the backward Euler method and the parametric bilinear transform [12]. In the case of linear time-invariant circuits, system discretization is equivalent to discretizing only the reactive circuit elements (e.g., inductor, capacitor) using that same method. Degrees of freedom in the discretization method can be used to alleviate some of the frequency response distortion. For simple circuits with only a single feature in the frequency response, an example of such approach is the parametric bilinear transform $[11,13]$ where the free parameter may be used to match the response at one frequency exactly. For circuits with a simple known pole/zero structure, the pole/zero frequencies themselves may be "pre-warped" to account for the anticipated frequency warping, for example in the case of graphical equalizers made out of cascades of second-order filters [14-16]. Another approach is to directly develop discretetime system whose parameters are selected to match properties from the original circuit, either using mapping equations [17-22], for example for graphical equalizers [23-25], or using optimization methods [26-28]. However, those approaches have limitations for complex circuits with wideband frequency response features, including the loss of correspondence with the original physical system (i.e., to the individual circuit elements), and/or the limited set of response features they target (e.g., Nyquist magnitude response, resonant and transition frequencies).

Recent work [13] showed how the use of differentiated discretization methods in a circuit could be leveraged to improve the match in driving point frequency response of audio circuits between the original and discretized systems over a wide range of frequencies without losing the structure of the underlying circuit, as each individual component remains attached to its own equation. That approach was illustrated using the example of the differentiated parametric bilinear transform. A different free parameter was set for each reactive element in the circuit through a joint optimization process aimed at minimizing the $L^{2}$ distance between the original and discretized frequency responses. Results showed a much closer match in terms of driving point frequency response over a wide frequency range compared to typical alternative approaches.

In this paper we extend the framework from [13] so that it can be used in the case where the output variable is an arbitrary combination of various circuit variables (i.e., node voltages, branch currents) and the case where there are multiple concurrent output variable of interest. We also present the $L^{1}$ norm as alternative to the $L^{2}$ norm for the error definition in the optimization process. Sec. 2 presents the mathematical framework of those extensions and Sec. 3 shows its successful application to the case of the vibrato/chorus circuit of the Hammond organ [29]. We use the same mathematical notation as outlined in [13].

\section{THEORY}

\subsection{General Tableau formulation}

Following a derivation similar to [13], we know that a circuit (or sub-circuit) with independent linear elements and a driving point 


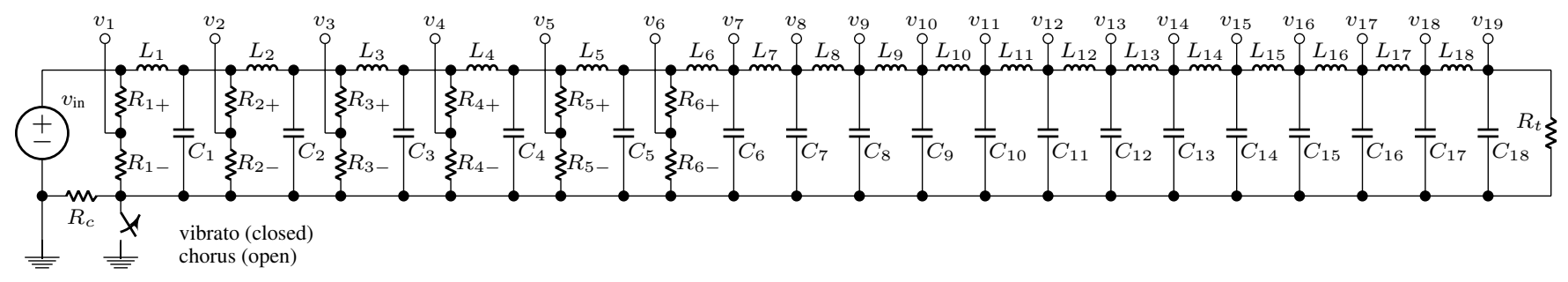

Figure 1: Vibrato/Chorus Schematic.

Table 1: Circuit component values.

\begin{tabular}{lrl}
\hline Name & value & units \\
\hline$R_{c}$ & 22 & $\mathrm{k} \Omega$ \\
$R_{1+}$ & 27 & $\mathrm{k} \Omega$ \\
$R_{1-}$ & 68 & $\mathrm{k} \Omega$ \\
$R_{2+}$ & 56 & $\mathrm{k} \Omega$ \\
$R_{3+}$ & 39 & $\mathrm{k} \Omega$ \\
$R_{2-}, R_{3-}$ & 0.15 & $\mathrm{M} \Omega$ \\
$R_{4+}$ & 33 & $\mathrm{k} \Omega$ \\
\hline
\end{tabular}

\begin{tabular}{lrl}
\hline Name & value & units \\
\hline$R_{5+}$ & 18 & $\mathrm{k} \Omega$ \\
$R_{6+}$ & 12 & $\mathrm{k} \Omega$ \\
$R_{4-} \ldots R_{6-}$ & 0.18 & $\mathrm{M} \Omega$ \\
$L_{1} \ldots L_{18}$ & 500 & $\mathrm{mH}$ \\
$C_{1} \ldots C_{17}$ & 0.004 & $\mu \mathrm{F}$ \\
$C_{18}$ & 0.001 & $\mu \mathrm{F}$ \\
$R_{t}$ & 15 & $\mathrm{k} \Omega$ \\
\hline
\end{tabular}

branch/port (whose voltage $u_{e}$ and current $i_{e}$ are tied through the branch equation $\alpha i_{e}-\beta u_{e}=e$ ) can be written as:

$$
\left[\begin{array}{ccc}
\mathbf{Z}_{L} & \mathbf{0}_{L} & -\mathbf{A}_{L}^{\top} \\
\mathbf{0}_{L}^{\top} & \alpha & -\beta \boldsymbol{a}_{e}^{\top} \\
\mathbf{A}_{L} & \boldsymbol{a}_{e} & \mathbf{0}_{N, N}
\end{array}\right]\left[\begin{array}{c}
\boldsymbol{i}_{L} \\
i_{e} \\
\boldsymbol{v}
\end{array}\right]=e\left[\begin{array}{c}
\mathbf{0}_{L} \\
1 \\
\mathbf{0}_{N}
\end{array}\right]
$$

with $\mathbf{A}_{L}$ the incidence matrix associated with the branches of the $L$ linear elements, $\mathbf{Z}_{L}$ the (diagonal) impedance matrix of the linear elements, $\boldsymbol{i}_{L}$ the branch currents for the linear elements, $e$ the driving point source value, $\boldsymbol{a}_{e}$ the incidence matrix of the voltage source branch, $i_{e}$ the branch current for the voltage source and $\boldsymbol{v}$ the voltages of the $N$ circuit nodes.

\subsection{Multi-output transfer function}

In [13], we derived the framework to optimize driving point transfer functions, i.e., transfer functions relating an input and an output both measured at the driving branch/port of the circuit. In this paper, we are interested in optimizing transfer functions in the more generic case of multiple output variables, i.e., where we have $M$ distinct output variables $o^{(1)}, . ., o^{(m)}, . ., o^{(M)}$ that are expressed as a linear combination of several circuit variables (node voltages, branch currents). Then, using matrix block inversion [30], we can obtain the expression of those variables from Eq. (1) as:

$$
\boldsymbol{o}=\left[\begin{array}{c}
o^{(1)} \\
\vdots \\
o^{(M)}
\end{array}\right]=\mathbf{G}\left[\begin{array}{c}
\boldsymbol{i}_{L} \\
i_{e} \\
\boldsymbol{v}
\end{array}\right]=\frac{e \mathbf{G}}{\alpha+\beta \theta}\left[\begin{array}{c}
-\mathbf{Y}_{L} \mathbf{A}_{L}^{\top} \boldsymbol{\Phi}^{-1} \boldsymbol{a}_{e} \\
1 \\
-\boldsymbol{\Phi}^{-1} \boldsymbol{a}_{e}
\end{array}\right]
$$

with $\mathbf{Y}_{L}=\mathbf{Z}_{L}^{-1}, \boldsymbol{\Phi}=\mathbf{A}_{L} \mathbf{Y}_{L} \mathbf{A}_{L}^{\top}, \theta=\boldsymbol{a}_{e}^{\top} \boldsymbol{\Phi}^{-1} \boldsymbol{a}_{e}$, and $\mathbf{G}$ a matrix of weights expressing the linear combination of circuit variables forming each output variable. The multi-output transfer function is then written as:

$$
\mathbf{H}(s)=\left[\begin{array}{c}
H^{(1)}(s) \\
\vdots \\
H^{(M)}(s)
\end{array}\right]=\frac{\boldsymbol{o}}{e}=\frac{\mathbf{G}}{\alpha+\beta \theta}\left[\begin{array}{c}
-\mathbf{Y}_{L} \mathbf{A}_{L}^{\top} \boldsymbol{\Phi}^{-1} \boldsymbol{a}_{e} \\
1 \\
-\boldsymbol{\Phi}^{-1} \boldsymbol{a}_{e}
\end{array}\right]
$$

\subsection{Discretization and transfer function derivatives}

Once we discretized the system, i.e., we discretize the admittance matrix $\mathbf{Y}_{L}$ as $\hat{\mathbf{Y}}_{L}$, we can form the discretized transfer function $\hat{\mathbf{H}}\left(z^{-1}\right)$ by replacing $\mathbf{Y}_{L}, \boldsymbol{\Phi}$ and $\theta$ by the discretized $\hat{\mathbf{Y}}_{L}, \hat{\boldsymbol{\Phi}}$ and $\hat{\theta}$ in Eq. (3). Another quantity of interest for the optimized discretization parameter selection is the partial derivatives of the transfer functions with respect to the discretization free parameter(s) $\psi$. From Eq. (3), these derivatives can be expressed as:

$$
\begin{aligned}
\frac{\partial \hat{\mathbf{H}}}{\partial \psi} & =\frac{\mathbf{G}}{\alpha+\beta \hat{\theta}}\left(\left[\begin{array}{c}
\mathbf{Y}_{L} \mathbf{A}_{L}^{\top} \boldsymbol{\Phi}^{-1} \mathbf{A}_{L}-\mathbf{I}_{L} \\
0 \\
\mathbf{\Phi}^{-1} \mathbf{A}_{L}
\end{array}\right] \frac{\partial \mathbf{Y}_{L}}{\partial \psi} \mathbf{A}_{L}^{\top} \boldsymbol{\Phi}^{-1} \boldsymbol{a}_{e}\right. \\
& \left.-\beta \frac{\boldsymbol{a}_{e}^{\top} \hat{\boldsymbol{\Phi}}^{-1} \mathbf{A}_{L} \frac{\partial \mathbf{Y}_{L}}{\partial \psi} \mathbf{A}_{L}^{\top} \hat{\boldsymbol{\Phi}}^{-1} \boldsymbol{a}_{e}}{\alpha+\beta \hat{\theta}}\left[\begin{array}{c}
\mathbf{Y}_{L} \mathbf{A}_{L}^{\top} \boldsymbol{\Phi}^{-1} \boldsymbol{a}_{e} \\
-1 \\
\boldsymbol{\Phi}^{-1} \boldsymbol{a}_{e}
\end{array}\right]\right)
\end{aligned}
$$

In our approach, we consider in particular the case where a given parameter $\psi$ is attached only to the $l$ th element, so that only its discretized admittance $\hat{y}_{l}$ depends on it, and as a consequence:

$$
\begin{aligned}
& \frac{\partial \mathbf{Y}_{L}}{\partial \psi}=\frac{\partial \hat{y}_{l}}{\partial \psi} \boldsymbol{\delta}_{l} \boldsymbol{\delta}_{l}^{\top} \quad \text { and } \\
& \frac{\partial \hat{\mathbf{H}}}{\partial \psi}=\frac{\mathbf{G}}{\alpha+\beta \hat{\theta}}\left(\boldsymbol{a}_{l}^{\top} \boldsymbol{\Phi}^{-1} \boldsymbol{a}_{e}\right) \frac{\partial \hat{y}_{l}}{\partial \psi}\left(\left[\begin{array}{c}
\mathbf{Y}_{L} \mathbf{A}_{L}^{\top} \boldsymbol{\Phi}^{-1} \boldsymbol{a}_{l}-\boldsymbol{\delta}_{l} \\
0 \\
\boldsymbol{\Phi}^{-1} \boldsymbol{a}_{l}
\end{array}\right]\right. \\
& \left.-\beta \frac{\boldsymbol{a}_{l}^{\top} \hat{\boldsymbol{\Phi}}^{-1} \boldsymbol{a}_{e}}{\alpha+\beta \hat{\theta}}\left[\begin{array}{c}
\mathbf{Y}_{L} \mathbf{A}_{L}^{\top} \boldsymbol{\Phi}^{-1} \boldsymbol{a}_{e} \\
-1 \\
\boldsymbol{\Phi}^{-1} \boldsymbol{a}_{e}
\end{array}\right]\right)
\end{aligned}
$$

where $\boldsymbol{a}_{l}$ (respectively $\boldsymbol{\delta}_{l}$ ) is the column of $\mathbf{A}_{L}$ (resp. $\mathbf{I}_{L}$ ) attached to the branch of the $l$ th element.

\subsection{Error description}

From the expression of the original transfer function value $\mathbf{H}_{\Omega}=$ $\mathbf{H}(j \Omega)$ at radian frequency $\Omega$ and the corresponding discretized transfer function value $\hat{\mathbf{H}}_{\Omega}=\hat{\mathbf{H}}\left(e^{j \Omega}\right)$, we want to select discretization parameters that minimize the system error similarly as in [13]. We choose to express the error $\epsilon$ to minimize as a vector norm over a given radian frequency range $\left.\left[\Omega_{1}, \Omega_{2}\right] \in\right] 0, \pi / T$, with:

$$
\epsilon=\|\mathbf{H}-\hat{\mathbf{H}}\|=\int_{\Omega_{1}}^{\Omega_{2}}\left\|\mathbf{H}_{\Omega}-\hat{\mathbf{H}}_{\Omega}\right\| d \Omega
$$




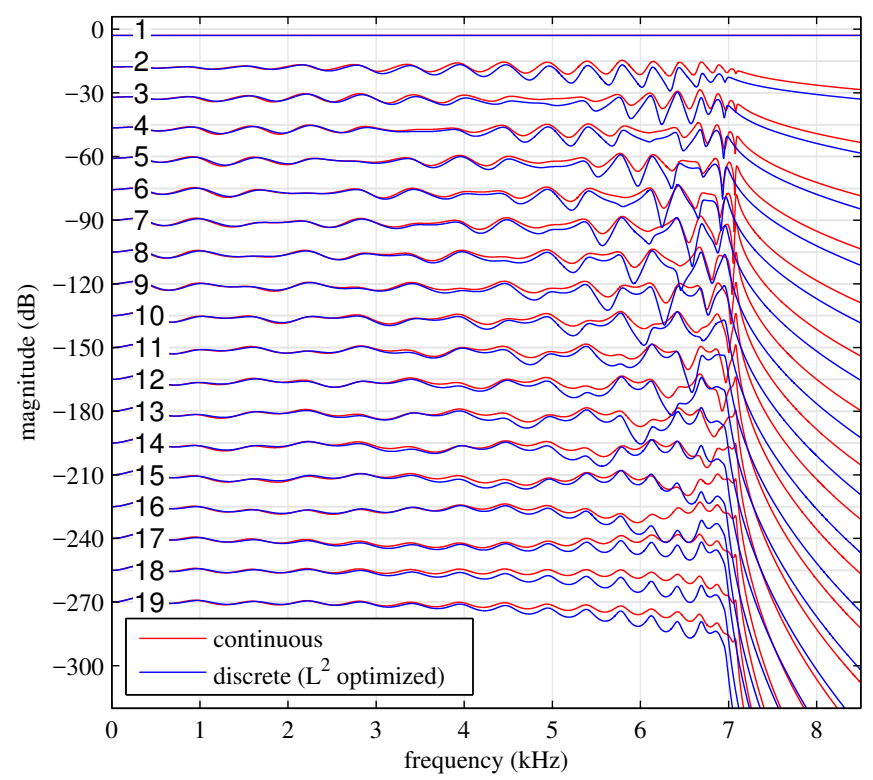

(a) $L^{2}$-optimized magnitude response.

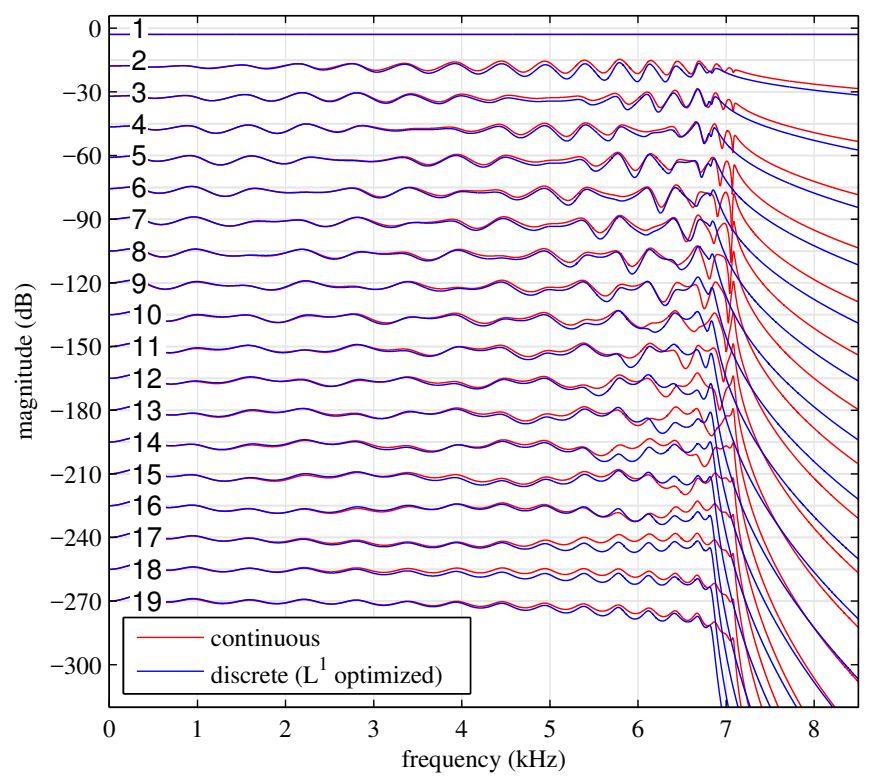

(b) $L^{1}$-optimized magnitude response.

Figure 2: Responses of the LC ladder at the 19 tap indices, using differentiated parametric bilinear transforms optimized using the $L^{2}$ and $L^{1}$ error functions. For readability, the taps are offset in $15 \mathrm{~dB}$ increments.

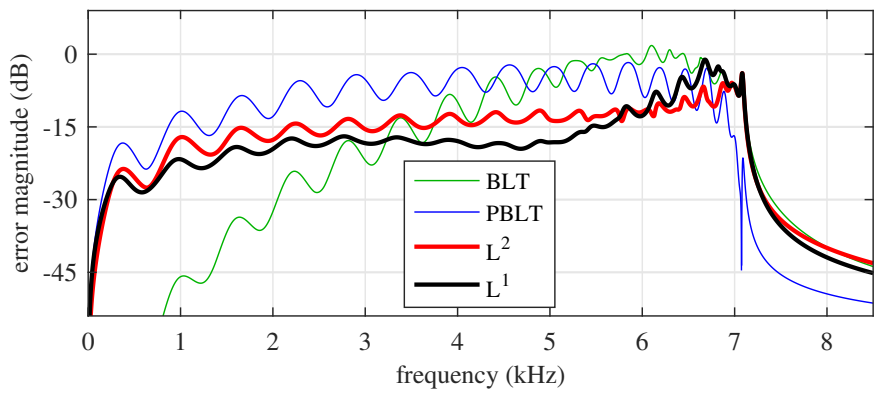

(a) Mean error magnitude $\left|H_{\Omega}^{(m)}-\hat{H}_{\Omega}^{(m)}\right|$.

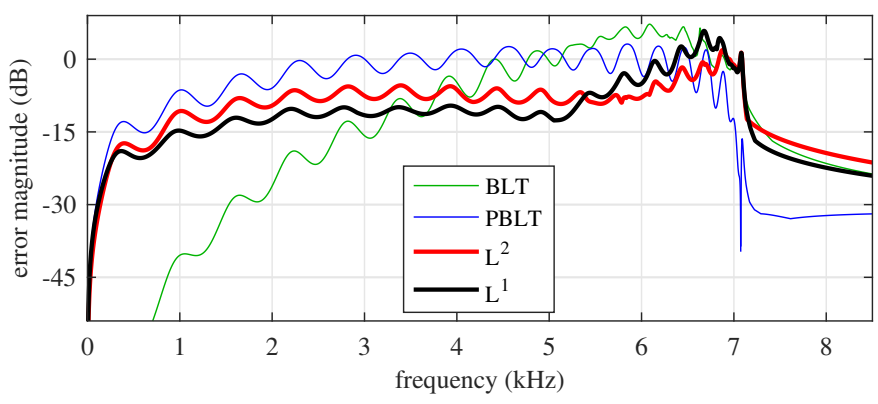

(b) Max error magnitude $\left|H_{\Omega}^{(m)}-\hat{H}_{\Omega}^{(m)}\right|$.

Figure 3: Mean and max error magnitudes across tap indices for the bilinear transform (BLT), the parametric bilinear transform (PBLT), and two differentiated parametric bilinear transform $\left(L^{2}\right.$-optimized and $L^{1}$-optimized).

Table 2: Jointly optimized differentiated $T^{\prime}$ coefficients. All are in units of $\mu$ s and rounded to five significant figures.

\begin{tabular}{cccccccccccccccccccccccc}
\hline norm & $m$ & 1 & 2 & 3 & 4 & 5 & 6 & 7 & 8 & 9 & 10 & 11 & 12 & 13 & 14 & 15 & 16 & 17 & 18 \\
\hline \multirow{2}{*}{$L^{2}$} & $L_{m}$ & 24.852 & 22.298 & 22.575 & 20.400 & 21.033 & 21.926 & 22.075 & 21.723 & 21.661 & 22.153 & 22.376 & 21.979 & 21.625 & 21.567 & 21.968 & 22.534 & 23.631 & 18.128 \\
& $C_{m}$ & 18.107 & 20.962 & 26.161 & 21.771 & 20.974 & 21.551 & 22.082 & 22.011 & 21.713 & 21.990 & 22.486 & 22.340 & 21.960 & 22.326 & 22.437 & 22.387 & 22.447 & 20.365 \\
\hline \multirow{2}{*}{$L^{1}$} & $L_{m}$ & 23.412 & 24.757 & 20.555 & 21.230 & 22.601 & 22.228 & 21.696 & 21.649 & 21.840 & 22.023 & 22.227 & 22.401 & 22.441 & 22.466 & 22.634 & 22.911 & 24.393 & 20.084 \\
& $C_{m}$ & 13.870 & 21.222 & 22.792 & 20.150 & 21.658 & 21.972 & 21.205 & 20.819 & 20.801 & 20.863 & 20.917 & 20.959 & 20.917 & 20.990 & 20.939 & 20.848 & 21.114 & 20.912 \\
\hline
\end{tabular}

As in [13], one possible choice for error description is the error function based on the $L^{2}$-norm which corresponds to:

$$
\epsilon_{L^{2}}=\|\mathbf{H}-\hat{\mathbf{H}}\|_{L^{2}}=\int_{\Omega_{1}}^{\Omega_{2}}\left(\mathbf{H}_{\Omega}^{*}-\hat{\mathbf{H}}_{\Omega}^{*}\right)^{\top}\left(\mathbf{H}_{\Omega}-\hat{\mathbf{H}}_{\Omega}\right) d \Omega
$$

and the error partial derivatives with respect to the discretization parameters $\psi$ (needed for the optimization algorithm) are:

$$
\frac{\partial \epsilon_{L^{2}}}{\partial \psi}=2 \int_{\Omega_{1}}^{\Omega_{2}} \operatorname{Re}\left[\left(\frac{\partial \hat{\mathbf{H}}^{*}}{\partial \psi}\right)^{\top}\left(\hat{\mathbf{H}}_{\Omega}-\mathbf{H}_{\Omega}\right)\right] d \Omega
$$

Optimizing the $L^{2}$ norm generally emphasizes a more even dis- 
tribution of the error across frequencies. An alternative error function is based on the $L^{1}$ norm. Compared to $L^{2}$, it generally emphasizes lower error values at most frequencies while concentrating larger error values at a few frequencies [31]. It is defined as:

$$
\epsilon_{L^{1}}=\| \mathbf{H}-\left.\hat{\mathbf{H}}\right|_{L^{1}}=\int_{\Omega_{1}}^{\Omega_{2}} \sum_{m=1}^{M}\left|H_{\Omega}^{(m)}-\hat{H}_{\Omega}^{(m)}\right| d \Omega
$$

and the error partial derivatives with respect to $\psi$ become:

$$
\frac{\partial \epsilon_{L^{1}}}{\partial \psi}=2 \int_{\Omega_{1}}^{\Omega_{2}} \operatorname{Re}\left[\sum_{m=1}^{M} \frac{\partial \hat{H}^{(m)}}{\partial \psi} \frac{\hat{H}_{\Omega}^{(m)}-H_{\Omega}^{(m)}}{\left|\hat{H}_{\Omega}^{(m)}-H_{\Omega}^{(m)}\right|}\right] d \Omega
$$

\subsection{Differentiated parametric bilinear transform}

As in [13], we consider in particular the case of the differentiated parametric bilinear transform. For linear circuits described in the $s$-domain, this discretization amounts to replacing instances of $s$ in each component's admittance $y_{l}(s)$ using the mapping:

$$
s \mapsto \frac{2}{T_{l}^{\prime}} \frac{1-z^{-1}}{1+z^{-1}}
$$

using a different bilinear transform parameter $T_{l}^{\prime}$ or each linear element to form a discretized admittance $\hat{y}_{l}\left(z^{-1}\right)$ so that:

$$
\begin{aligned}
\hat{y}_{l}\left(z^{-1}\right) & =y_{l}\left(\frac{2}{T_{l}^{\prime}} \frac{1-z^{-1}}{1+z^{-1}}\right) \quad \text { and } \\
\frac{\partial \hat{y}_{l}}{\partial T_{n}^{\prime}}\left(z^{-1}\right) & = \begin{cases}-\frac{2}{T_{l}^{\prime 2}} \frac{\partial y_{l}}{\partial s}\left(\frac{2}{T_{l}^{\prime}} \frac{1-z^{-1}}{1+z^{-1}}\right) & \text { if } l=n, \\
0 & \text { otherwise. }\end{cases}
\end{aligned}
$$

\section{CASE STUDY}

\subsection{Circuit description}

We apply the framework described above to the design of differentiated discretization for the LC ladder section of the Hammond organ vibrato/chorus effect circuit [29]. The ladder section is a 19-stage ladder structure terminated by a resistor (see Fig. 1 and component values in Tab. 1). We focus here on the vibrato setting of the circuit, so that the resistor $R_{c}$ can be ignored. In terms of input/output structure, this circuit feeds the second part of the system, the scanner device, which scans successively various subsets of the taps $v_{m}$ ( $m=1 \ldots 19)$. As such, the scanner section essentially performs an interpolation between two of the node voltages [29]. As input, the circuit is driven by the voltage value of the source $v_{\text {in }}$.

\subsection{Optimization}

Following the circuit description, the relevant circuit variables to observe and optimize correspond to the 19 node voltages $v_{m}(m=$ $1 \ldots 19$ ) indicated in Fig. 1. We thus set the $\mathbf{G}$ matrix so that each of the node voltages is set as one of the output variables $o^{(m)}$ to optimize. As described in [29], the transfer function associated with each of the node voltages in the original system corresponds to a low-pass filter with a cutoff at roughly $7075 \mathrm{~Hz}$ whose passband contains a complex pattern of ripples. Furthermore, neither the response associated with the system discretized using the standard bilinear transform nor the one associated with the system discretized using the parametric bilinear transform for which $T^{\prime}$ was chosen to match the responses at the cutoff frequency $7075 \mathrm{~Hz}$ properly match the transfer functions in the passband. Differentiated parametric bilinear transforms are then optimized using our approach to generate a system that matches better the different transfer functions.

\subsection{Implementation}

To perform the optimization, we use an implementation of the circuit coded in MATLAB 2016b using integral [32] with default options to perform numerical integration for Eq. (7) and fmincon $[33,34]$ to perform the iterative error minimization with the known analytical gradient expression, the constraints $T_{l}^{\prime}>0$ for all $m$ and default options. The sampling frequency is set at $48 \mathrm{kHz}$. The optimization range is between $20 \mathrm{~Hz}$ and $20 \mathrm{kHz}$ roughly matching the human auditory range. The initial solution for the optimization is set as the bilinear transform solution $T_{l}^{\prime}=20.833 \mu$ s for all $\mathrm{m}$.

\subsection{Results}

We show the differentiated coefficients $T^{\prime}$ obtained optimizing the $L^{2}$ and $L^{1}$ error functions in Tab. 2. They can be compared to the bilinear transform solution $\left(T_{l}^{\prime}=20.833 \mu \mathrm{s}\right)$ and to the parametric bilinear transform for which the cutoff frequency response is matched $\left(T_{l}^{\prime}=22.462 \mu \mathrm{s}\right)$. The magnitude response corresponding to the 19 outputs is shown in Fig. 2a (respectively Fig. 2b) for the $L^{2}$ (resp. $L^{1}$ ) error function along with the original continuous-time system response. Those figures can be compared to the ones in [29] that show the responses in the case of the bilinear transform and the parametric bilinear transform. We show in Fig. 3a (respectively Fig. 3b) the mean (resp. the maximum) value of $\left|H_{\Omega}^{(m)}-\hat{H}_{\Omega}^{(m)}\right|$ across frequencies for the four approaches. As expected for the bilinear transform, the error grows with frequency due to the warping distortion, with a rather large error on the upper half of the passband. The parametric bilinear transform matches best the response at the cutoff frequency at the expense of a much larger overall error in the passband. Finally, we see that both optimized methods significantly lower and spread the error across the passband, with the exception of some residual error around the cutoff frequency. In more detail, the $L^{2}$ optimization matches more closely the cutoff, while the $L^{1}$ optimization lowers more significantly the error across most of the the passband. We can then select the best approach depending on the desired trade-off for the discretized system.

\section{CONCLUSION}

In this paper, we presented an extension of the framework presented in [13], aiming at using differentiated discretization schemes across the reactances of audio circuits in order for the discretized system to more closely match the original frequency response. This extension goes beyond driving point transfer functions, adding the general case where the output variable depends on an arbitrary combination of the circuit variables, and where multiple output variables need to be considered at once, and considering error metrics based on the $L^{1}$ and the $L^{2}$ norms for the optimization. We detailed the process to express the optimization problem associated with the differentiated discretization parameter selection starting from the Tableau formulation of the studied circuit. Finally, we validated this framework by successfully improving the discretization of the LC ladder section of the Hammond organ vibrato/chorus circuit, a complex audio circuit with wideband frequency response features and 19 simultaneous output variables, compared to typical approaches. 


\section{REFERENCES}

[1] E. Süli and D. Mayers, An introduction to numerical analysis. Cambridge, UK: Cambridge Univ. Press, 2003.

[2] P. Moin, Fundamentals of engineering numerical analysis. New York, NY: Cambridge Univ. Press, 2010.

[3] J. Vlach and K. Singhal, Computer methods for circuit analysis and design. New York, NY: Van Nostrand Reinhold Company, 1983.

[4] G. De Sanctis and A. Sarti, "Virtual analog modeling in the wave-digital domain," IEEE Trans. Audio, Speech, Language Process., vol. 18, no. 4, pp. 715-27, 2010.

[5] A. Fettweis, "Wave digital filters: Theory and practice," Proc. IEEE, vol. 74, no. 2, pp. 270-327, 1986.

[6] K. Werner, J. Smith, and J. Abel, "Wave digital filter adaptors for arbitrary topologies and multiport linear elements," in Proc. 18th Int. Conf. Digital Audio Effects, Trondheim, Norway, 2015.

[7] K. Werner, V. Nangia, J. Smith, and J. Abel, "Resolving wave digital filters with multiple/multiport nonlinearities," in Proc. 18th Int. Conf. Digital Audio Effects, Trondheim, Norway, 2015.

[8] K. Werner, "Virtual analog modeling of audio circuitry using wave digital filters," Ph.D. diss., Stanford Univ., Stanford, CA, 2016.

[9] D. Yeh, J. Abel, and J. Smith, "Automated physical modeling of nonlinear audio circuits for real-time audio effects-part I: Theoretical development," IEEE Trans. Audio, Speech, Language Process., vol. 18, no. 4, pp. 728-37, 2010.

[10] M. Holters and U. Zölzer, "A generalized method for the derivation of non-linear state-space models from circuit schematics," in Proc. 23rd European Signal Process. Conf., Nice, France, 2015.

[11] J. Smith, Physical audio signal processing. W3K Pub., 2010.

[12] F. Germain and K. Werner, "Design principles for lumped model discretisation using Möbius transforms," in Proc. 18th Int. Conf. Digital Audio Effects, Trondheim, Norway, 2015.

[13] — , "Joint parameter optimization of differentiated discretization schemes for audio circuits," in Proc. 142 Conv. Audio Eng. Soc. (AES), Berlin, Germany, 2017.

[14] J. Abel and D. Berners, "Filter design using second-order peaking and shelving sections," in Proc. Int. Conf. Comput. Music (ICMC), Miami, FL, 2004.

[15] Z. Chen, G. Geng, F. Yin, and J. Hao, "A pre-distortion based design method for digital audio graphic equalizer," Digital Signal Process., vol. 25, pp. 296-302, 2014

[16] V. Välimäki and J. Reiss, "All about audio equalization: Solutions and frontiers," Appl. Sci., vol. 6, no. 5, 2016.

[17] L. Rabiner and B. Gold, Theory and application of digital signal processing. Englewood Cliffs, NJ: Prentice Hall, 1975.
[18] T. Parks and C. Burrus, Digital filter design. New York, NY: John Wiley, 1987.

[19] M. A. Al-Alaoui, "Novel stable higher order s-to-z transforms," IEEE Trans. Circuits Syst. I, Fundam. Theory Appl., vol. 48, no. 11, pp. 1326-9, 2001.

[20] T. Šekara, "New transformation polynomials for discretization of analogue systems," Elect. Eng., vol. 89, no. 2, pp. 137-47, 2006.

[21] T. Stilson, "Efficiently-variable non-oversampled algorithms in virtual-analog music synthesis," Ph.D. diss., Stanford Univ., Stanford, CA, 2006.

[22] M. Al-Alaoui, "Al-Alaoui operator and the new transformation polynomials for discretization of analogue systems," Elect. Eng., vol. 90, no. 6, pp. 455-67, 2008.

[23] S. Orfanidis, "Digital parametric equalizer design with prescribed Nyquist-frequency gain," J. Audio Eng. Soc, vol. 45, no. 6, pp. 444-55, 1997.

[24] D. Berners and J. Abel, "Discrete-time shelf filter design for analog modeling," in Proc. 115 Conv. Audio Eng. Soc. (AES), New York, NY, 2003.

[25] M. Massberg, "Digital low-pass filter design with analogmatched magnitude response," in Proc. 131 Conv. Audio Eng. Soc. (AES), New York, NY, 2011.

[26] J. Markel and A. Gray, Linear prediction of speech. New York, NY: Springer, 1976, vol. 12.

[27] E. Maestre, G. Scavone, and J. Smith, "Design of recursive digital filters in parallel form by linearly constrained pole optimization," IEEE Signal Process. Lett., vol. 23, no. 11, pp. 1547-50, 2016.

[28] _ - "Joint modeling of bridge admittance and body radiativity for efficient synthesis of string instrument sound by digital waveguides," IEEE/ACM Trans. Audio, Speech, Language Process., vol. 25, no. 5, pp. 1128-39, 2017.

[29] K. Werner, W. Dunkel, and F. Germain, "A computational model of the Hammond organ vibrato/chorus using wave digital filters," in Proc. 19th Int. Conf. Digital Audio Effects, Brno, Czech Republic, 2016.

[30] F. Zhang, The Schur complement and its applications. New York, NY: Springer, 2005.

[31] S. Boyd and L. Vandenberghe, Convex optimization. Cambridge, UK: Cambridge Univ. Press, 2004.

[32] L. Shampine, "Vectorized adaptive quadrature in MATLAB," J. Comput. Appl. Math., vol. 211, no. 2, pp. 131-40, 2008.

[33] R. Byrd, J. Gilbert, and J. Nocedal, "A trust region method based on interior point techniques for nonlinear programming," Math. Prog., vol. 89, no. 1, pp. 149-85, 2000.

[34] R. Waltz, J. Morales, J. Nocedal, and D. Orban, "An interior algorithm for nonlinear optimization that combines line search and trust region steps," Math. Prog., vol. 107, no. 3, pp. 391408, 2006. 